\title{
SOX4 arrests lung development in rats with hyperoxia-induced bronchopulmonary dysplasia by controlling EZH2 expression
}

\author{
BINGTING PAN, XINDONG XUE, DAN ZHANG, MENGYUN LI and JIANHUA FU \\ Department of Pediatrics, Shengjing Hospital of China Medical University, Shenyang, Liaoning 110004, P.R. China
}

Received September 4, 2016; Accepted September 21, 2017

DOI: 10.3892/ijmm.2017.3171

\begin{abstract}
Bronchopulmonary dysplasia (BPD) is currently the most common severe complication in premature infants and is characterized by the arrest of alveolar and vascular growth. Alveolar type II cells play an important role in the pathological foundation of BPD. An association of BPD with epithelial-to-mesenchymal transition (EMT) in type II cells exposed to hyperoxia was previously identified. SOX4, a transcription factor that is indispensable to embryogenesis, including lung development, participates in regulating EMT and cell survival, affecting tumorigenesis. The aim of the present study was to investigate the involvement of SOX4 in the occurrence of BPD, which, to the best of our knowledge, has not been previously determined. For this purpose, newborn rats were randomly divided into two treatment groups: The model group was exposed to hyperoxia $\left(80-85 \% \mathrm{O}_{2}\right)$, while the control group was kept under normoxic conditions $\left(21 \% \mathrm{O}_{2}\right)$. Lung tissues were collected on postnatal days 1, 3, 7, 14 and 21 and morphological changes in the lungs were examined by hematoxylin and eosin staining. The location of SOX4 in type II cells was detected by double immunofluorescence. The expression of SOX 4 and enhancer of zeste homolog 2 (EZH2) in type II cells and lung tissues were detected by immunochemistry, western blotting and quantitative polymerase chain reaction analysis. The results demonstrated that, compared with the control group, the radial alveolar count decreased rapidly in the model group, accompanied by increased mean alveolar diameter and alveolar septal thickness. SOX4 and EZH2 were highly expressed in type II cells exposed to hyperoxia. However, in total lung tissues, SOX4 and EZH2 expression was profoundly decreased in the early stages and increased in the late stages following exposure to hyperoxia. The expression of the EZH2 protein was positively correlated with that of the SOX4 protein. In conclusion, at the alveolar stage, which is a critical period after birth for lung development,
\end{abstract}

Correspondence to: Professor Jianhua Fu, Department of Pediatrics, Shengjing Hospital of China Medical University, 36 Sanhao Street Shenyang, Liaoning 110004, P.R. China

E-mail: fujh@sj-hospital.org

Key words: SOX4, enhancer of zeste homolog 2, epithelial-tomesenchymal transition, bronchopulmonary dysplasia, lung development hyperoxia induced dysregulation of SOX4 and EZH2 in rat lungs, indicating that SOX4 may contribute to the disruption of lung development in BPD by regulating EZH2 expression.

\section{Introduction}

Bronchopulmonary dysplasia (BPD) is a common chronic lung disease in premature infants. With the introduction of prenatal steroid use, progressive ventilator strategies, and improved survival of very low birth weight (VLBW) infants, the incidence of BPD has increased to as high as $68 \%$ among infants born at 22-28 weeks of gestation (1). These infants require re-admission to the hospital within the first 2 years after birth, and even as adolescents for lung function abnormalities consistent with increased incidence of asthma, chest deformities and pulmonary hypertension. Additionally, delayed neurodevelopment, particularly cerebral palsy, cognitive and motor function abnormalitites, are common in BPD infants; however, there is yet no effective and safe therapy for this condition (2).

'Old BPD', associated with prominent fibroproliferation, which was first characterized by Northway et al in 1967, is currently less striking. The more modern concept is that BPD is a disruption of distal lung growth, with prominent impaired alveolar and vascular growth, which has been defined as 'new BPD' (2). Mechanical ventilation and oxygen supplementation are major contributors to BPD (3). Unlike 'old BPD', patients with 'new BPD' may have been exposed to low inspired oxygen concentrations (2). Hyperoxia-induced inflammation, oxidative stress, apoptosis and cell death lead to disruption of lung development (4).

Epithelial-to-mesenchymal transition (EMT) is generally known as the process during which epithelial cells lose their polarity and junctions, and acquire the characteristics of mesenchymal cells (5). It was previously confirmed that hyperoxia induced type II cells in the lung to differentiate into fibroblasts through EMT, thus affecting alveolar development (6). This is associated with downregulation of RUNX3, a gene that is crucial for lung development. It was also demonstrated that the EMT occurring in the BPD model is associated with epigenetic modifications. Histone methyltransferase enhancer of zeste homolog 2 (EZH2), a key epigenetic modification regulator, was upregulated in type II cells in the model group and silenced the expression of RUNX3 through trimethylated H3K27 (7). SOX4, which belongs to the SRY-related HMG box gene family (8), is a master regulator during transforming 
growth factor (TGF)- $\beta$-induced EMT (9). More recently, selected studies have elucidated the role of the SOX4/EZH2 axis in epigenetic modifications during EMT $(10,11)$. The aim of the present study was to determine whether dysregulated expression of SOX4 in the hyperoxia-exposed lung may contribute to EMT in BPD through EZH2.

\section{Materials and methods}

Animal model. All animal procedures were reviewed and approved by the Experimental Animal Ethics Committee of China Medical University. A total of 20 pregnant Sprague-Dawley rats (age, 8-10 weeks; weight, 200-250 g) were purchased from the Experimental Animal Center of China Medical University, and the BPD model was constructed as previously described. Briefly, newborn rats were randomly divided into two groups within $12 \mathrm{~h}$ of birth: The BPD model rats were exposed to $80-85 \%$ oxygen in a sealed Plexiglas tank for 1-21 days, while the control group rats were exposed to room air (21\% oxygen). During this time, the oxygen concentration was continuously monitored with an oxygen analyzer and maternal rats were switched between the model and control groups every $24 \mathrm{~h}$ to avoid oxygen toxicity.

Lung tissue specimens. On postnatal days 1, 3, 7, 14 and 21, 8 pups in each group were anesthetized by intraperitoneal injection with $10 \%$ chloral hydrate, and the lungs were inflated with phosphate-buffered saline (PBS) at $20 \mathrm{~cm} \mathrm{H}_{2} \mathrm{O}$ pressure; the lung tissues were then collected. The right middle lung lobes were fixed in 4\% paraformaldehyde (PFA) for hematoxylin and eosin (H\&E) and immunohistochemical staining; the remaining lung lobes were stored at $-80^{\circ} \mathrm{C}$ for subsequent quantitative PCR (qPCR) and western blot analysis.

Lung histology. PFA-fixed lung tissues were dehydrated through graded alcohol and xylene, and embedded in paraffin. Subsequently, $4-\mu \mathrm{m}$ sections were prepared for H\&E staining for morphometric analysis. The radial alveolar count (RAC) provides a simple and relatively accurate assessment of lung development. According to the radial count method proposed by Emery and Mithal (12), alveoli transected along the perpendicular line from the center of the respiratory bronchiole to the nearest connective tissue septum or pleura, are enumerated. Image-Pro Plus 6.0 software (Media Cybernetics, Rockville, MD, USA) was used to measure the mean alveolar diameter (MAD) and alveolar septal thickness (AST). At least 5 fields were captured per section, and at least 20 alveoli were measured per field.

Double immunofluorescence. Following drying in a $60^{\circ} \mathrm{C}$ oven overnight, the tissue sections were deparaffinized in xylene and rehydrated in a gradient ethanol series. For antigen retrieval, the sections were microwaved in $10 \mathrm{mM}$ citrate buffer $(\mathrm{pH}$ 6.0) for $20 \mathrm{~min}$. The sections were then incubated in $5 \%$ bovine serum for $1 \mathrm{~h}$ at $37^{\circ} \mathrm{C}$. A combination of two primary antibodies, rabbit polyclonal anti-SOX4 (SAB2108306; 1:100 dilution; Sigma-Aldrich Merck KGaA, St. Louis, MO, USA) and mouse monoclonal anti-p180 (ab24751; 1:50 dilution; Abcam, Cambridge, UK), was used for incubating the sections overnight at $4^{\circ} \mathrm{C}$, while for negative controls PBS was used in the place of the antibody. The sections were then incubated with a mixture of two secondary antibodies, Alexa Fluor-594 donkey anti-rabbit IgG (ab150076; 1:500 dilution; Abcam) and Alexa Fluor-488 donkey anti-mouse IgG (ab150105; 1:500 dilution; Abcam) for $4 \mathrm{~h}$ at room temperature, followed by DAPI incubation for nuclear staining. Double immuofluoresence images were acquired using a confocal laser-scanning microscope (C1; Nikon, Tokyo, Japan).

Immunohistochemistry. Following deparaffinization and microwave heating, as previously described, the sections were treated sequentially with $3 \% \mathrm{H}_{2} \mathrm{O}_{2}$ for 20 min to block endogenous peroxidase activity, and goat serum for $1 \mathrm{~h}$ at $37^{\circ} \mathrm{C}$ to block non-specific binding. The sections were incubated overnight at $4^{\circ} \mathrm{C}$ with SOX4 antibody (SAB2108306; 1:1500 dilution; Sigma-Aldrich) or EZH2 antibody (612666; 1:200 dilution; BD Transduction Laboratories, San Jose, CA, USA), then incubated with secondary antibody and streptavidin-horseradish peroxidase for $20 \mathrm{~min}$. Finally, the sections were developed with 3,3'-diaminobenzidine and counterstained with hematoxylin. Antigen expression was assessed using an image analyzer (Image-Pro Plus 6.0; Media Cybernetics, Rockville, MD, USA).

Western blotting. Western blotting was performed using standard protocols. In brief, total protein was extracted from frozen lung tissue samples and mixed with loading buffer. Equal amounts of protein were separated by $10 \%$ sodium dodecyl sulfate-polyacrylamide gel electrophoresis (SDA-PAGE) (100 V for $3 \mathrm{~h}$ ) and transferred to polyacrylamide difluoride (PVDF) membranes (100 V for $50 \mathrm{~min}$ ). Membranes were then blocked in 5\% skimmed milk for $2 \mathrm{~h}$ at room temperature. Membranes incubated with rabbit anti-SOX4 (SAB2108306; 1:800 dilution; Sigma-Aldrich Merck KGaA) or mouse anti-EZH2 (612666; 1:1,000 dilution; BD Transduction Laboratories, San Jose, CA, USA) were shaken overnight at $4^{\circ} \mathrm{C}$. The next day, the membranes were incubated with horseradish peroxidase-conjugated goat antirabbit or anti-mouse secondary antibody (1:5,000 dilution; Proteintech, Rosemont, IL, USA) for $2 \mathrm{~h}$ after washing three times in Tris-buffered saline and Polysorbate 20, and developed using enhanced chemiluminescence reagents (Thermo Scientific Pierce; Thermo Fisher Scientific, Waltham, MA, USA). Densitometry values were detected for all bands and standardized relative to glyceraldehyde 3-phosphate dehydrogenase (GAPDH) for each sample.

$q P C R$. Total RNA was extracted from the right lung lobes using TRIzol reagent (Takara Biotechnology Co., Kyoto, Japan) according to the manufacturer's instructions. Following purification according to the kit instructions, cDNA was reverse-transcribed from $1 \mu \mathrm{g}$ RNA from each sample using Prime Script RT reagent kit with gDNA Eraser (Takara Biotechnology Co.). The primers were designed as follows: SOX4 forward, 5'-ATGTCCCTGGGCAGTTTCAG-3' and reverse, 5'-TGCAATAGTCCGGGAACTCG-3'; GAPDH forward, 5'-AGACAGCCGCATCTTCTTGT-3' and reverse, 5'-CTTGCCGTGGGTAGAGTCAT-3'. qPCR was performed using SYBR Premix Ex Tag (Takara Biotechnology Co.) on LightCycler (Thermo Fischer Scientific, Carlsbad, CA, USA); 
A
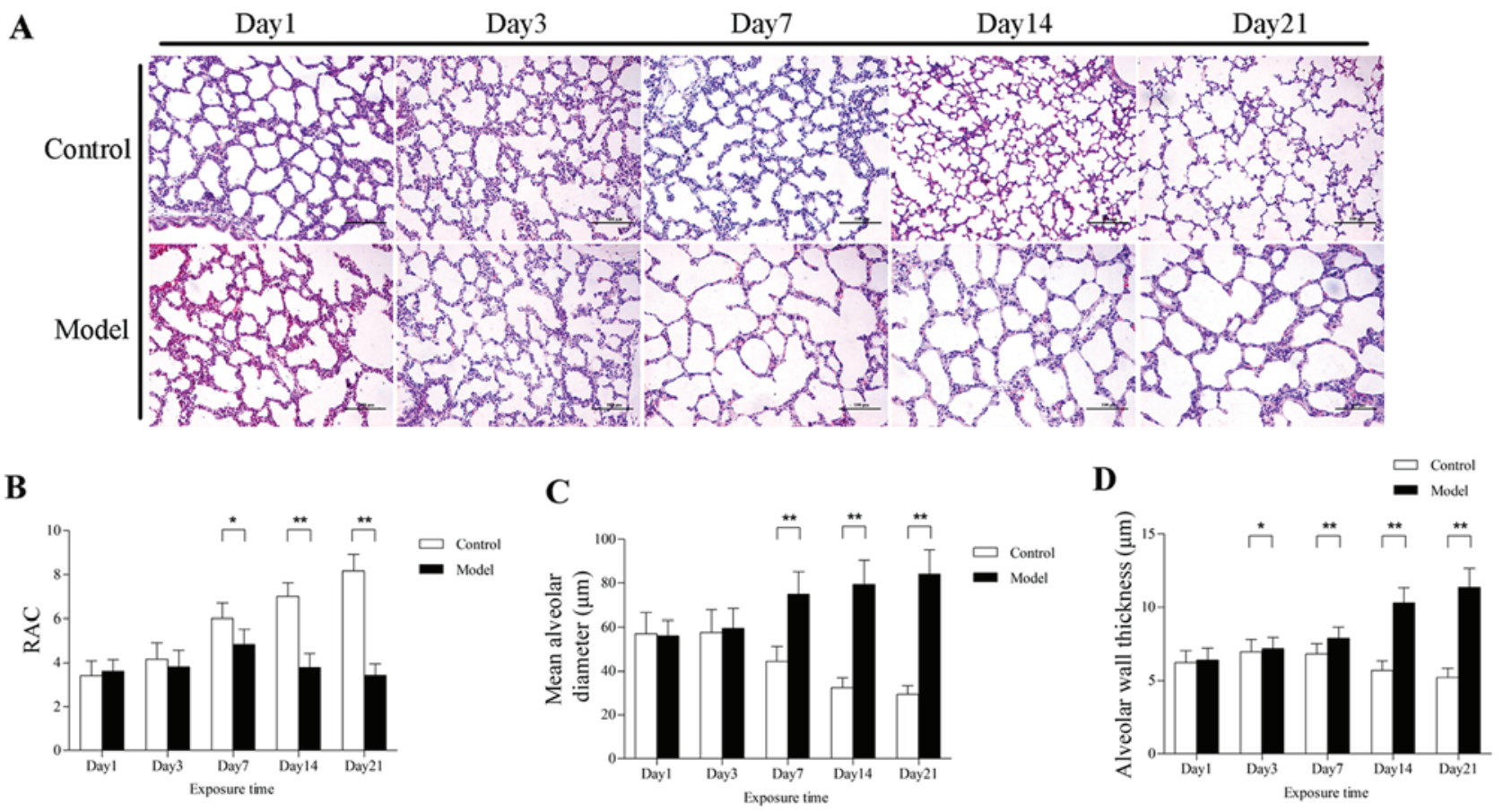

Figure 1. Morphological changes in rat lungs. (A) Hematoxylin and eosin staining of rat lungs exposed to hyperoxia (model) or normoxia (control). Scale bar, $100 \mu \mathrm{m}$ (B) Compared with the control rats, the radial alveolar counts (RACs) of model rats were significantly reduced on days 7, 14 and 21 . (C and D) After exposure to hyperoxia for $\geq 7$ days, $(\mathrm{C})$ the mean alveolar diameter and (D) the alveolar septal thickness increased significantly. ${ }^{*} \mathrm{P}<0.05$ and ${ }^{* *} \mathrm{P}<0.01$.

the amplification program was $95^{\circ} \mathrm{C}$ for $30 \mathrm{sec}, 40$ cycles of $95^{\circ} \mathrm{C}$ for $30 \mathrm{sec}$ and $60^{\circ} \mathrm{C}$ for $34 \mathrm{sec}$. Relative mRNA expression of SOX4 normalized to GAPDH was determined by the $2^{-\Delta \Delta \mathrm{Cq}}$ method.

Statistical analysis. Statistical analysis was performed using SPSS 21.0 software (IBM, Armonk, NY, USA). Any significant difference between two groups was compared using an unpaired t-test, and correlation analysis was performed via Pearson's tests. All data are presented as mean \pm standard deviation. $\mathrm{P}<0.05$ was considered to indicate statistically significant differences.

\section{Results}

Prolonged hyperoxia disrupts lung development. Compared with the control group, the survival rate of rat pups exposed to hyperoxia was significantly decreased. As an important index of lung development, the RACs of the control group increased gradually, beginning on the day of birth. In pups exposed to hyperoxia for days, the RACs were significantly lower compared with those of the control group, particularly on days 7, 14 and 21 (Fig. 1B). Additionally, prolonged hyperoxia caused substantial morphological alteration of lung tissues. There was an increase in alveolar size in the model group, as quantified by an increase in MAD and AST, and the difference between the two groups was more pronounced with the prolongation of the hyperoxia exposure time (Fig. 1C and D). By contrast, no significant histological difference was found on days 1 and 3. Taken together, these results demonstrate that prolonged exposure to hyperoxia interrupts alveolarization, as demonstrated by a decreased number of alveoli and increased diameter and septal thicknesses of the alveoli.
SOX4 expression in alveolar type II cells exposed to hyperoxia. Double immunofluorescence was used to determine SOX4 localization. p180 is a lamellar body protein that is highly expressed in the lung, and plays an important role in the formation of pulmonary surfactant. Green p180 fluorescence represented type II cells. Co-expression of p180 and red SOX4 fluorescence appeared as orange. In the control group, only few cells co-expressed red SOX4 and green p180 fluorescence. By contrast, cells with red SOX4 fluorescence in the nuclei and green p180 fluorescence or orange co-expressed fluorescence in the cytoplasm were observed in the model group (Fig. 2).

SOX4 expression in rat lungs exposed to hyperoxia. To investigate whether hyperoxia altered the expression of SOX4 in rat lungs, immunohistochemistry, western blot analysis and qPCR were performed. After hyperoxia exposure for $\geq 7$ days, SOX4 was localized to the nucleus as well as the cytoplasm of alveolar epithelial cells in the model group, but was poorly expressed in the control group (Fig. 3). The results differed, however, in total lung tissues. On day 1, the level of SOX4 expression was not different between the model and control groups, at both the mRNA and protein level. Furthermore, SOX4 protein expression was found to be downregulated after hyperoxia exposure for 3 days $(\mathrm{P}<0.05)$ and decreased markedly at 7 and 14 days $(\mathrm{P}<0.01)$. When hyperoxia exposure was prolonged to 21 days, the SOX4 protein expression in the model group, rather than decreasing, it was higher compared with that under normoxic conditions $(\mathrm{P}<0.01)$ (Fig. 4A and B). A similar effect on SOX4 mRNA expression was verified by qPCR analysis, but there was no significant difference at 21 days (Fig. 4C).

EZH2 expression in rat lungs exposed to hyperoxia. EZH2 was expressed in the nuclei of alveolar epithelial cells. After 

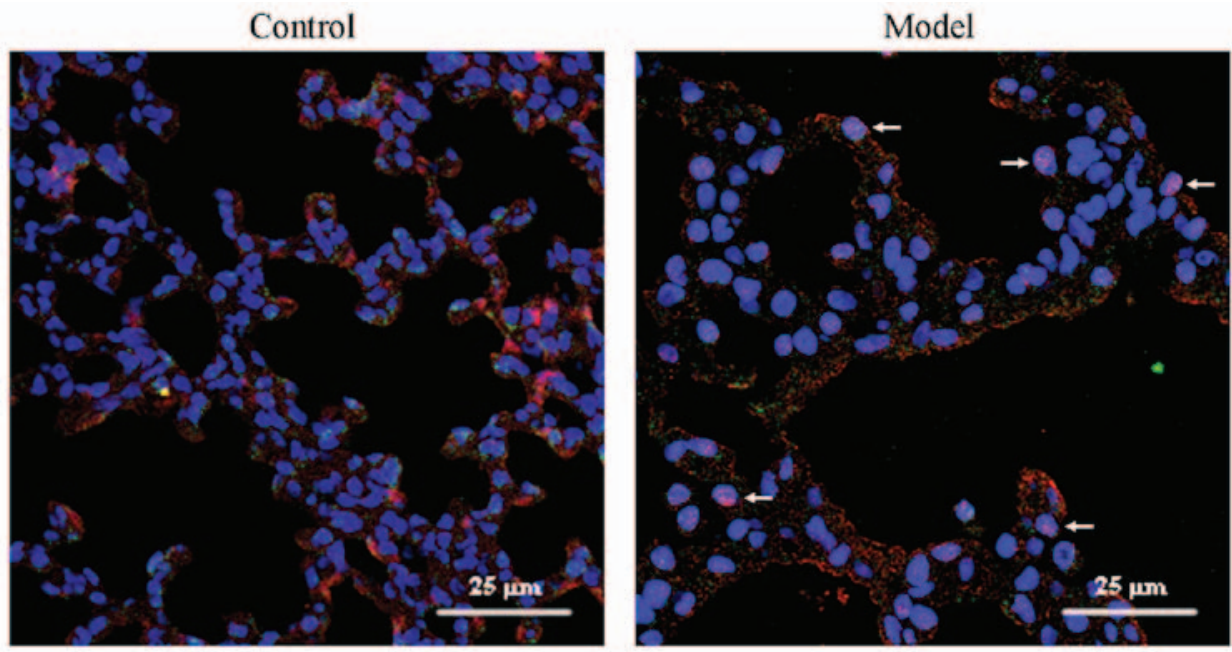

Figure 2. Co-expression of SOX4 and p180 in type II cells. Green fluorescence represented p180 expression, red fluorescence represented SOX4 expression, and orange fluorescence represented co-expression of SOX 4 and p180. In the control group, type II cells co-expressing p180 and SOX4 were not identified. In the model group, SOX4 was expressed in the nucleus as well as in the cytoplasm, and co-expression of SOX4 and p180 was observed in type II cells. Scale bar, $25 \mu \mathrm{m}$.

A

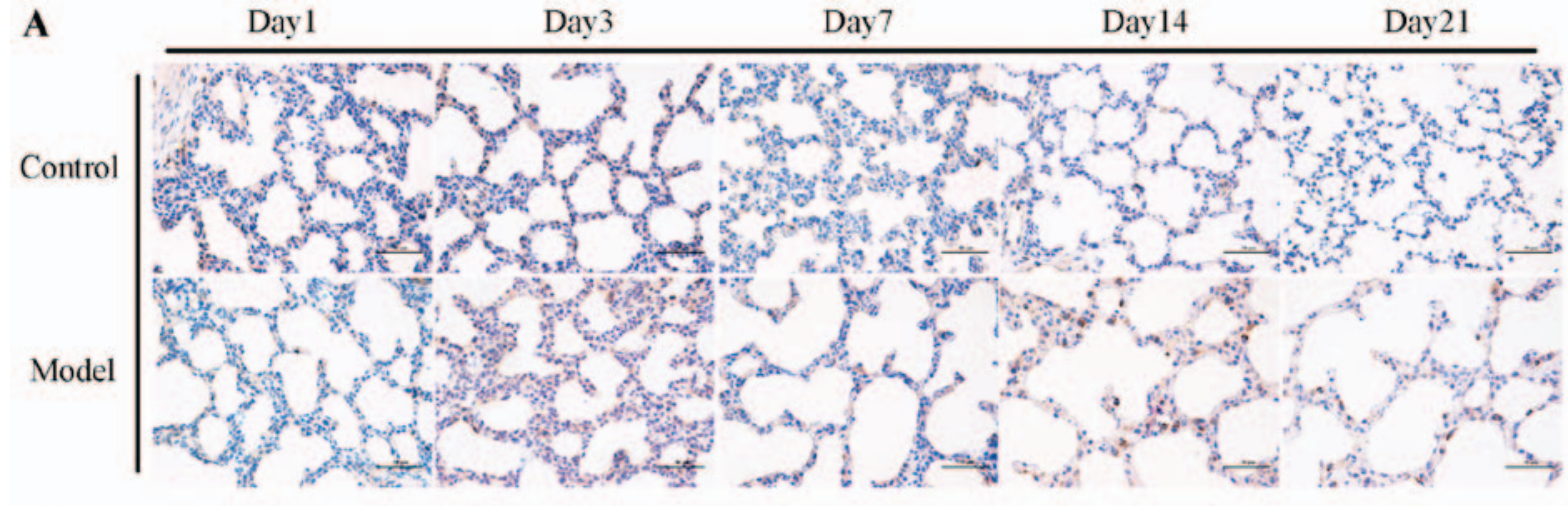

Day 7

Day14

Day21

B

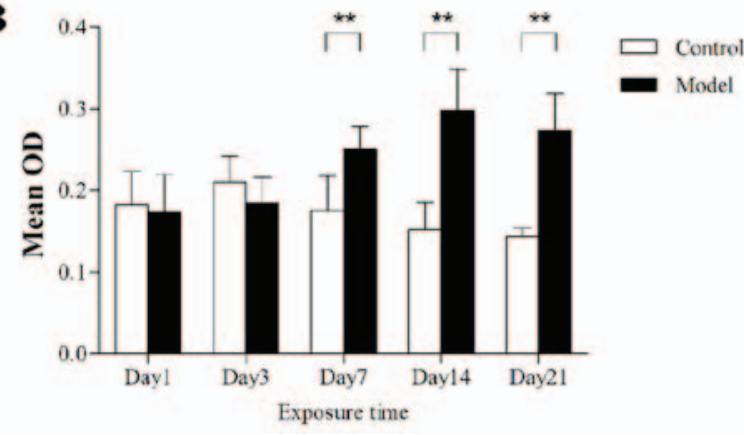

Figure 3. SOX4 expression in type II cells in the control and model groups. (A) SOX4 was expressed in the nucleus as well as the cytoplasm of type II cells, which were mainly concentrated at the alveolar septal tips. Scale bar, $50 \mu \mathrm{m}$ (B) Analyses of mean ODs of SOX4-positive cells. SOX4 expression increased in the model group beginning on day 7 . $\mathrm{P}<0.05$ and ${ }^{* *} \mathrm{P}<0.01$. OD, optical density.

exposure to hyperoxia for 14 and 21 days, EZH2 expression was markedly upregulated $(\mathrm{P}<0.01)$ (Fig. 5A and $\mathrm{C})$. However, the EZH2 protein levels in total lung tissues were downregulated in the model group at 7 and 14 days, as demonstrated by western blot analysis $(\mathrm{P}<0.01)$. EZH2 protein expression in the model group, compared with that in the control group, was increased at 21 days (Fig. 5B and D).

Correlation between SOX4 expression and EZH2 in rat lungs. To confirm the correlation between SOX4 protein expression and EZH2, correlation analyses were performed. EZH2 protein expression was found to be positively correlated with that of SOX4 (r=0.87; P<0.01) (Fig. 6).

\section{Discussion}

The etiology of BPD is multifactorial, and long-term mechanical ventilation is one of the primary risk factors increasing the incidence of BPD in VLBW infants, although it may decrease mortality in this patient population (13). In the present study, lower oxygen concentrations were used (80-85\%) compared with those in previous studies (90-95\%), and it was demonstrated that 
$\mathbf{A}$

$$
\frac{\text { Day1 }}{\mathrm{c} m} \frac{\mathrm{Day} 3}{\mathrm{c} m} \frac{\mathrm{Day} 7}{\mathrm{c} m} \frac{\mathrm{Day} 14}{\mathrm{c} m} \frac{\text { Day21 }}{\mathrm{c} m}
$$

SOX 4

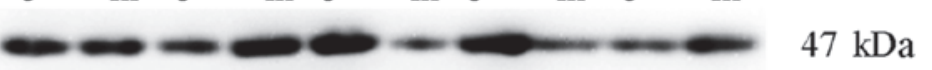

GAPDH

$36 \mathrm{kDa}$
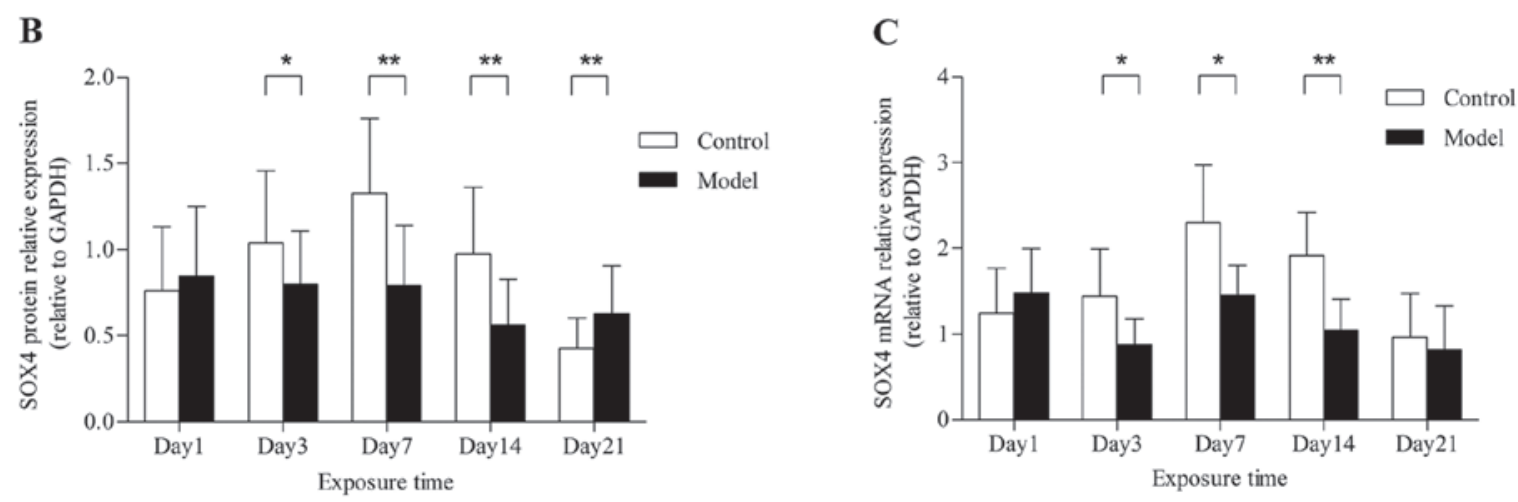

Figure 4. SOX4 expression changes in total lung tissues. (A and B) SOX4 protein levels in the model group decreased rapidly on days 3,7 and 14, and increased on day 21. (C) The SOX4 mRNA levels also decreased after exposure to hyperoxia for 3, 7 and 14 days, but there was no difference between the model and control groups on day $21 .{ }^{*} \mathrm{P}<0.05$ and ${ }^{* *} \mathrm{P}<0.01$.

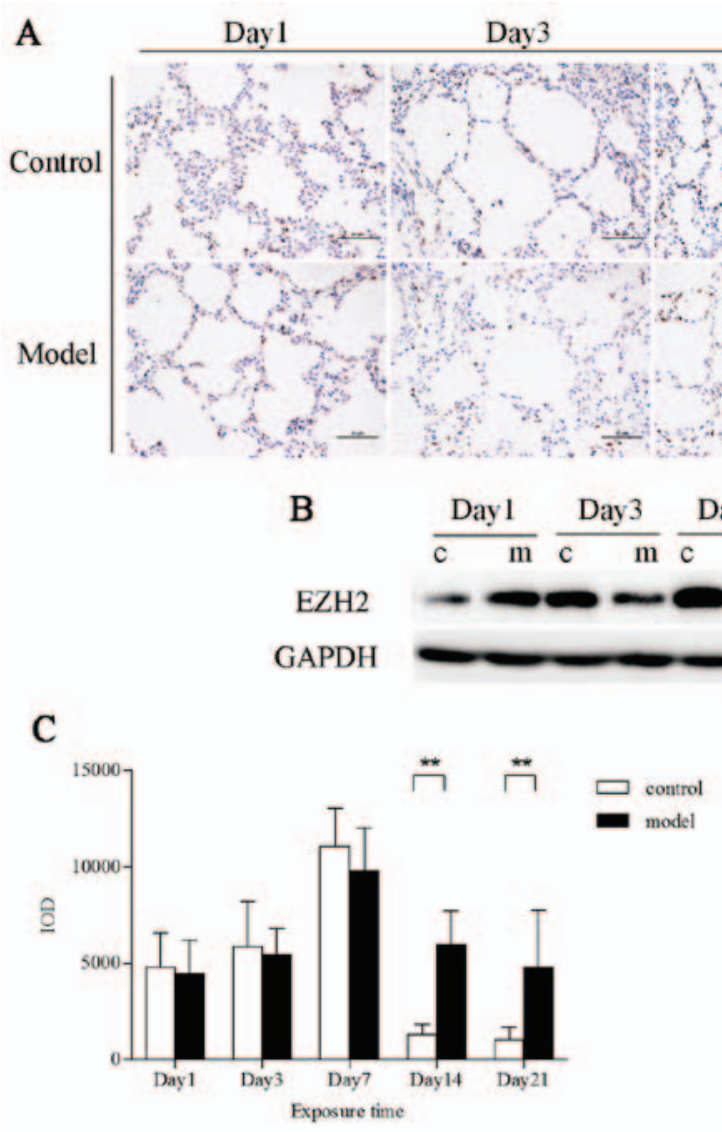

Day7 Day14

Day21
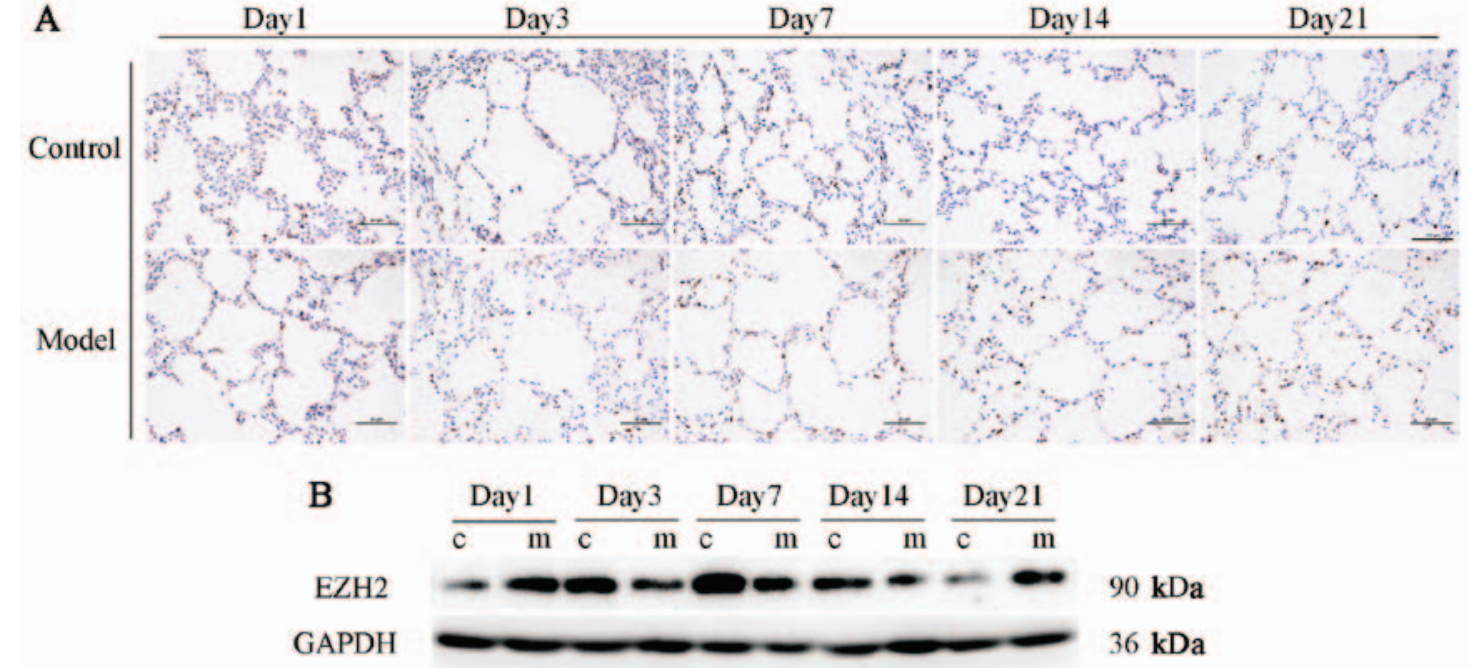

C
D

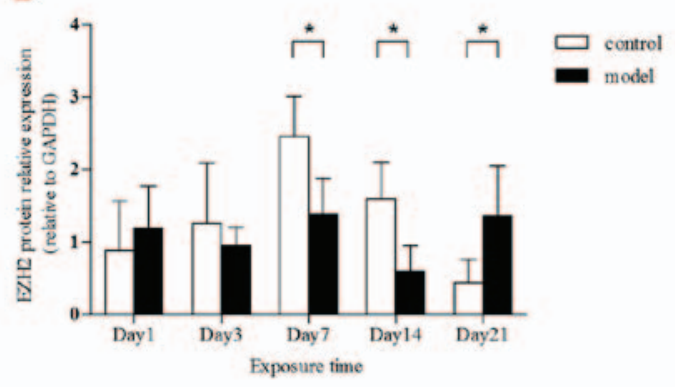

Figure 5. EZH2 expression changes in the lung. (A and C) EZH2 was expressed in the nuclei of type II cells. EZH2-positive cells were analyzed by integrated optical density (IOD). EZH2 expression in type II cells was increased at 14 and 21 days in the model group (P<.01). Scale bar, $50 \mu \mathrm{m}$. (B and C) Compared with the control group, EZH2 expression in total lung tissues was decreased in the model group at 7 and 14 days $(\mathrm{P}<0.05)$. Exposure to hyperoxia for 21 days increased EZH2 expression $(\mathrm{P}<0.05)$. ${ }^{*} \mathrm{P}<0.05$ and ${ }^{* *} \mathrm{P}<0.01$. EZH2, enhancer of zeste homolog 2.

prolonged hyperoxia inhibited pulmonary development. There was a distinct morphological change: The alveoli decreased in number and were larger, more simplified, and displaying thickened alveolar septa in the model group, consistently with the pathological characteristics of 'new BPD'. The underlying mechanisms, however, remain largely unknown (2). 


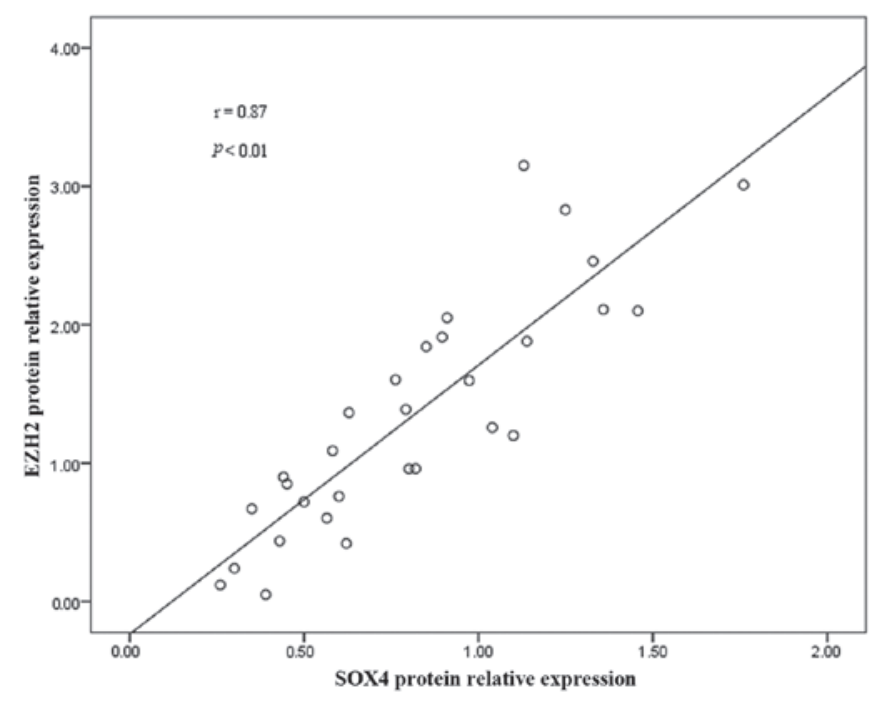

Figure 6. Correlation analyses between SOX4 expression and EZH2 in rat lungs. SOX4 protein expression was positively correlated with that of EZH2; $\mathrm{r}=0.87, \mathrm{P}<0.01 . \mathrm{EZH} 2$, enhancer of zeste homolog 2 .

The alveolar epithelial surface is composed of alveolar type I and II cells. Type II cells comprise $16 \%$ of total lung cells and $60 \%$ of alveolar epithelial cells, which only cover $5 \%$ of the alveolar surface (14). Regeneration of the alveolar epithelium following lung injury is one of the major functions attributed to alveolar type II cells. Upon exposure to hyperoxia, type I cells are more susceptible to injury, and type II cells appear to increasingly proliferate and transdifferentiate into type I cells, which are resistant to oxidative damage (15). However, previous studies confirmed that hyperoxia inhibits activation of type II cells $(16,17)$. A number of studies on the pathological foundation of BPD have focused on type II cell injury, with respect to proliferation, apoptosis and transdifferentiation into type I cells or mesenchymal cells (EMT).

EMT is accompanied by apparent changes in cell morphology and motility. As a physiological phenomenon, EMT is involved in embryogenesis, tumorigenesis and wound healing through cell signaling pathways, such as TGF- $\beta /$ Smad and Wnt/ $\beta$-catenin (18). It was previously demonstrated that alveolar type II cells underwent EMT in hyperoxia-induced BPD in newborn rats. Ultrastructural changes occurred in type II cells that differentiated into fibroblasts, followed by altered expression of E-cadherin, $\mathrm{N}$-cadherin and $\alpha$-smooth muscle actin (6). RUNX3 is a runt-domain transcription factor that plays a crucial role in lung development. In RUNX3 null mouse lungs, EMT markers are abnormally expressed, along with low levels of E-cadherin and high fibronectin expression (19). Our previous study confirmed that RUNX3 is downregulated in the hyperoxia-induced BPD model, which is attributable to EZH2-catalyzed histone methylation (7). EZH2, the histone methyltransferase, is a component of polycomb-repressive complex 2 (20). EZH2 epigenetically silences gene expression through the trimethylation of histone 3 lysine 27 (H3K27me3), and has been implicated in several types of cancer (11). However, the mechanism of EZH2 expression regulation in BPD is entirely unknown.
SOX4, a member of the group C subfamily of SOX transcription factors, plays an important role in embryogenesis and cell fate determination (21). SOX4 is important for lung development. Its expression is widespread in both the epithelium and the mesenchyme of the developing lung (22), with its expression in the bronchial duct epithelium being more abundant compared with that in surrounding lung tissues (23). During the canalicular period, SOX4 participates in transcription during differentiation of the conducting airway epithelium and affects lung morphogenesis (24). Its abnormal expression may cause various lung diseases. In non-small-cell lung cancer, SOX4 is overexpressed through $6 \mathrm{p}$ gene amplification and plays an important role in cancer progression and prognosis $(25,26)$. The present study demonstrated that SOX4 expression increased during the early postnatal stages and decreased in the later stages in the control group, with lower expression in type II cells and higher expression in total lung tissues compared with the BPD group. The low proportion of type II cells in rat lung tissues, of which $46 \%$ and $30 \%$ are capillary endothelial and interstitial cells, respectively (14), may account for this result. This suggests that SOX4 dysregulation may be one of the causes of BPD.

SOX4 is a master regulator during TGF- $\beta$-induced EMT(9). In breast cancer, SOX4 is overexpressed and triggers EMT to promote tumor progression and metastasis (27). Ablation of SOX4 expression results in reduced expression of EMT inducers, such as Snail, Twist and Zeb (28). In the present study, SOX4 was upregulated in type II cells and may be associated with the occurrence of EMT in the BPD model. More recently, certain studies elucidated the importance of the SOX4/EZH2 axis in epigenetic modification during EMT $(10,11)$. SOX4 directly regulates the expression of $\mathrm{EZH} 2$, which is involved in gene silencing via $\mathrm{H} 3 \mathrm{~K} 27 \mathrm{me} 3$ modification in the promoters of target genes such as the epithelial marker E-cadherin $(10,29)$. This study also revealed that EZH2 was upregulated in type II cells exposed to hyperoxia, and increased at a later time compared with SOX4. EZH2 expression increased, beginning 14 days after hyperoxia inhalation, while SOX4 increased after 7 days. Correlation analysis between SOX4 and EZH2 protein expression revealed a positive correlation. According to these findings, we hypothesized that upregulation of SOX4 in type II cells may promote EMT by modulating EZH2 expression, thereby disrupting alveolarization and lung development in BPD.

As lower expression of SOX4 in total lung tissues was observed in the model group, SOX 4 may act through a different mechanism in other lung cells, such as fibroblasts. It was previously demonstrated that prolonged hyperoxia results in high BAX expression in fibroblasts and promotes apoptosis (30). Another study demonstrated that downregulated SOX4 expression induces apoptosis in lung cancer (31). Hur et al reported that the HMG box of SOX4 interacts with p53 and reduces p53 transcriptional activity on the BAX promoter, resulting in inhibition of p53-mediated apoptosis (32). Therefore, reduced SOX4 expression in lung tissues may relieve its inhibition of p53-mediated apoptosis and upregulate BAX expression, thereby promoting apoptosis of lung fibroblasts in the model group. Additionally, it has been confirmed that repressing $\mathrm{EZH} 2$ inhibits proliferation and induces apoptosis in cancer 
cells (33). Consistent with SOX4 expression, the expression of EZH2 in total lungs in the model group was also reduced; however, this reduction occurred later compared with that of SOX4, indicating that SOX4 may promote fibroblast apoptosis by regulating EZH2.

As EMT generates fibroblasts for wound healing and tissue regeneration (17), upregulated expression of SOX4 in type II cells may trigger epithelial cells to transdifferentiate into fibroblasts during oxidative epithelial injury repair. Downregulation of SOX 4 in the early stages of hyperoxia in fibroblasts appeared to restrain the process of EMT against excessive fibroblast proliferation, and increased SOX4 expression in the later stages may indicate severe EMT that may lead to fibrosis, thus adversely affecting long-term prognosis. This hypothesis, which is consistent with lineage tracing studies showing that the number of fibroblasts transdifferentiated from epithelial cells is small, despite co-expression of epithelial and mesenchymal markers during human idiopathic pulmonary fibrosis (34), may be closer to the definition of the 'new BPD'. Otherwise, EMT of type II cells may interfere with their transdifferentiation into type I cells, leading to abnormal epithelial injury repair and, thus, affecting alveolarization. A study recently reported that SOX4 induction may be inhibited by caffeine, which has been used for BPD management (35). Thus, SOX4 may be a novel therapeutic target for BPD. Using caffeine during the early stages of BPD may downregulate SOX4 expression in the later stages, improving long-term prognosis.

In conclusion, the present study confirmed that the expression of the transcription factor SOX4 is increased in type II cells in BPD, but is reduced in total lung tissues. Dysregulation of SOX4 disrupts lung development in BPD by controlling EZH2 expression. However, further studies are required to determine the precise mechanism of action of SOX4 in BPD.

\section{Acknowledgements}

The present study was supported by grants from the Natural Science Foundation of China (nos. 81571479 and 81471489).

\section{References}

1. Baker CD and Abman SH: Impaired pulmonary vascular development in bronchopulmonary dysplasia. Neonatology 107: 344-351, 2015.

2. Kinsella JP, Greenough A and Abman SH: Bronchopulmonary dysplasia. Lancet 367: 1421-1431, 2006.

3. Baraldi E and Filippone M: Chronic lung disease after premature birth. N Engl J Med 357: 1946-1955, 2007.

4. Bhandari V: Hyperoxia-derived lung damage in preterm infants. Semin Fetal Neonatal Med 15: 223-229, 2010.

5. Lamouille S, Xu J and Derynck R: Molecular mechanisms of epithelial-mesenchymal transition. Nat Rev Mol Cell Biol 15: 178-196, 2014.

6. Yang H, Fu J, Xue X, Yao L, Qiao L, Hou A, Jin L and Xing Y: Epithelial-mesenchymal transitions in bronchopulmonary dysplasia of newborn rats. Pediatr Pulmonol 49: 1112-1123, 2014.

7. Zhu Y, Fu J, Yang H, Pan Y, Yao L and Xue X: Hyperoxiainduced methylation decreases RUNX3 in a newborn rat model of bronchopulmonary dysplasia. Respir Res 16: 75, 2015.

8. Wegner M; M W: From head to toes: The multiple facets of Sox proteins. Nucleic Acids Res 27: 1409-1420, 1999.

9. Vervoort SJ, van Boxtel R and Coffer PJ: The role of SRY-related HMG box transcription factor 4 (SOX4) in tumorigenesis and metastasis: Friend or foe? Oncogene 32: 3397-3409, 2013.
10. Tiwari N, Tiwari VK, Waldmeier L, Balwierz PJ, Arnold P, Pachkov M, Meyer-Schaller N, Schübeler D, van Nimwegen E and Christofori G: Sox 4 is a master regulator of epithelial-mesenchymal transition by controlling Ezh2 expression and epigenetic reprogramming. Cancer Cell 23: 768-783, 2013.

11. Hasegawa S, Nagano H, Konno M, Eguchi H, Tomokuni A, Tomimaru Y, Asaoka T, Wada H, Hama N, Kawamoto K, et al: A crucial epithelial to mesenchymal transition regulator, Sox4/Ezh2 axis is closely related to the clinical outcome in pancreatic cancer patients. Int J Oncol 48: 145-152, 2016.

12. Emery JL and Mithal A: The number of alveoli in the terminal respiratory unit of man during late intrauterine life and childhood. Arch Dis Child 35: 544-547, 1960.

13. Jobe AH: The new bronchopulmonary dysplasia. Curr Opin Pediatr 23: 167-172, 2011.

14. Crapo JD, Barry BE, Gehr P, Bachofen M and Weibel ER: Cell number and cell characteristics of the normal human lung. Am Rev Respir Dis 126: 332-337, 1982.

15. Castranova V, Rabovsky J, Tucker JH and Miles PR: The alveolar type II epithelial cell: A multifunctional pneumocyte. Toxicol Appl Pharmacol 93: 472-483, 1988.

16. Hou A, Fu J, Yang H, Zhu Y, Pan Y, Xu S and Xue X: Hyperoxia stimulates the transdifferentiation of type II alveolar epithelial cells in newborn rats. Am J Physiol Lung Cell Mol Physiol 308: L861-L872, 2015.

17. Xu W, Xu B, Zhao Y, Yang N, Liu C, Wen G and Zhang B: Wnt5a reverses the inhibitory effect of hyperoxia on transdifferentiation of alveolar epithelial type II cells to type I cells. J Physiol Biochem 71: 823-838, 2015.

18. Pain M, Bermudez O, Lacoste P, Royer PJ, Botturi K, Tissot A, Brouard S, Eickelberg O and Magnan A: Tissue remodelling in chronic bronchial diseases: from the epithelial to mesenchymal phenotype. Eur Respir Rev 23: 118-130, 2014.

19. Lee JM, Shin JO, Cho KW, Hosoya A, Cho SW, Lee YS, Ryoo HM, Bae SC and Jung HS.: Runx3 is a crucial regulator of alveolar differentiation and lung tumorigenesis in mice. Differentiation 81: 261-268, 2011.

20. Wang C, Liu X, Chen Z, Huang H, Jin Y, Kolokythas A, Wang A, Dai Y, Wong DT and Zhou X: Polycomb group protein EZH2-mediated E-cadherin repression promotes metastasis of oral tongue squamous cell carcinoma. Mol Carcinog 52: 229-236, 2013.

21. Penzo-Méndez AI: Critical roles for SoxC transcription factors in development and cancer. Int J Biochem Cell Biol 42: 425-428, 2010.

22. Dy P, Penzo-Méndez A, Wang H, Pedraza CE, Macklin WB and Lefebvre V: The three SoxC proteins - Sox4, Sox11 and Sox12 - exhibit overlapping expression patterns and molecular properties. Nucleic Acids Res 36: 3101-3117, 2008.

23. Hoser M, Potzner MR, Koch JM, Bösl MR, Wegner M and Sock E: Sox 12 deletion in the mouse reveals nonreciprocal redundancy with the related Sox 4 and Sox11 transcription factors. Mol Cell Biol 28: 4675-4687, 2008.

24. Maeda Y, Davé V and Whitsett JA: Transcriptional control of lung morphogenesis. Physiol Rev 87: 219-244, 2007.

25. Wang D, Hao T, Pan Y, Qian X and Zhou D: Increased expression of SOX4 is a biomarker for malignant status and poor prognosis in patients with non-small cell lung cancer. Mol Cell Biochem 402: 75-82, 2015.

26. Medina PP, Castillo SD, Blanco S, Sanz-Garcia M, Largo C, Alvarez S, Yokota J, Gonzalez-Neira A, Benitez J, Clevers HC, et al: The SRY-HMG box gene, SOX4, is a target of gene amplification at chromosome $6 \mathrm{p}$ in lung cancer. Hum Mol Genet 18: 1343-1352, 2009

27. Vervoort SJ, Lourenço AR, van Boxtel R and Coffer PJ: SOX4 mediates TGF- $\beta$-induced expression of mesenchymal markers during mammary cell epithelial to mesenchymal transition. PLoS One 8: e53238, 2013.

28. Parvani JG and Schiemann WP: Sox4, EMT programs, and the metastatic progression of breast cancers: Mastering the masters of EMT. Breast Cancer Res 15: R72, 2013.

29. Cao Q, Yu J, Dhanasekaran SM, Kim JH, Mani RS, Tomlins SA, Mehra R, Laxman B, Cao X, Yu J, et al: Repression of E-cadherin by the polycomb group protein EZH2 in cancer. Oncogene 27: 7274-7284, 2008.

30. Hu Y, Liu X, Zhang H, Fu J and Xue X: dynamic changes of $\mathrm{Bax} / \mathrm{Bcl}-2$ expression in lung tissue and fibroblasts of neonatal rats after inhaling high concentration oxygen. J Appl Clin Pediatr 26: 589-592, 2011. 
31. Zhou Y, Wang X, Huang Y, Chen Y, Zhao G, Yao Q, Jin C, Huang Y, Liu X and Li G: Downregulated SOX4 expression suppresses cell proliferation, metastasis and induces apoptosis in Xuanwei female lung cancer patients. J Cell Biochem 116 1007-1018, 2015.

32. Hur W, Rhim H, Jung CK, Kim JD, Bae SH, Jang JW, Yang JM, Oh ST, Kim DG, Wang HJ, et al: SOX4 overexpression regulates the p53-mediated apoptosis in hepatocellular carcinoma: Clinical implication and functional analysis in vitro. Carcinogenesis 31 : 1298-1307, 2010.

33. Xie L, Zhang Z, Tan Z, He R, Zeng X, Xie Y, Li S, Tang G, Tang $\mathrm{H}$ and He X: MicroRNA-124 inhibits proliferation and induces apoptosis by directly repressing EZH2 in gastric cancer. Mol Cell Biochem 392: 153-159, 2014.
34. Kage $\mathrm{H}$ and Borok Z: EMT and interstitial lung disease: A mysterious relationship. Curr Opin Pulm Med 18: 517-523, 2012.

35. Pan X, Zhao J, Zhang WN, Li HY, Mu R, Zhou T, Zhang HY, Gong WL, Yu M, Man JH, et al: Induction of SOX4 by DNA damage is critical for p53 stabilization and function. Proc Natl Acad Sci USA 106: 3788-3793, 2009.

(i) $\Theta$ This work is licensed under a Creative Common Attribution-NonCommercial-NoDerivatives 4.0 International (CC BY-NC-ND 4.0) License. 